\title{
Stereotactic Body Radiotherapy for Hepatocellular Carcinoma: Current Evidence and the Feasibility of Radiomics-based Predictive Models
}

\section{Yang-Hong Dai}

Tri-Service General Hospital

Po-Chien Shen

Tri-Service General Hospital

\section{Wei-Chou Chang}

Tri-Service General Hospital

Chen-Hsiang Lo

Tri-Service General Hospital

Jen-Fu Yang

Tri-Service General Hospital

Chun-Shu Lin

Tri-Service General Hospital

Hsing-Lung Chao

Tri-Service General Hospital

Chieh-Sheng Lu

Kaohsiung Armed Forces General Hospital

Shu-Ju Tu

Chang Gung University

Wen-Yen Huang ( $\sim$ hwyyi@yahoo.com.tw )

Tri-Service General Hospital https://orcid.org/0000-0002-8354-3320

\section{Research}

Keywords: radiation therapy, hepatocellular carcinoma, radiomics, machine learning

Posted Date: March 12th, 2020

DOI: https://doi.org/10.21203/rs.3.rs-17027/v1

License: (a) This work is licensed under a Creative Commons Attribution 4.0 International License.

Read Full License 


\section{Abstract}

Background : Stereotactic body radiotherapy (SBRT) is an effective but less focused alternative for treatment of hepatocellular carcinoma (HCC). To date, a personalized model for predicting therapeutic response is lacking. This study aimed to review current knowledge and to propose a radiomics-based machine-learning $(\mathrm{ML})$ strategy for local response (LR) prediction.

Methods : We searched the literature for studies conducted between January 1993 and August 2019 that used $>100$ patients. Additionally, $172 \mathrm{HCC}$ patients in our hospital were retrospectively analyzed between January 2007 and December 2016. In the radiomic analysis, 41 treated tumors were contoured and 46 radiomic features were extracted.

Results : The 1-year local control was $85.4 \%$ in our patient cohort, comparable with current results (87$99 \%$ ). The Support Vector Machine (SVM) classifier, based on computed tomography (CT) scans in the A phase processed by equal probability (Ep) quantization with 8 gray levels, showed the highest mean F1 score (0.7995) for favorable LR within 1 year (W1R), at the end of follow-up (EndR), and condition of infield failure-free (IFFF). The area under the curve (AUC) for this model was $92.1 \%, 96.3 \%$, and $99.2 \%$ for W1R, EndR, and IFFF, respectively.

Conclusions : SBRT has high 1-year local control and our study sets the basis for constructing predictive models for HCC patients receiving SBRT.

\section{Background}

In recent decades, stereotactic body radiotherapy (SBRT) has emerged as an attractive option for tumor ablation, including in hepatocellular carcinoma (HCC) $[1,2]$. However, SBRT is still usually reserved for salvage therapy in patients who are unsuitable or refractory to other locoregional therapies, probably due to the lack of randomized controlled trials and reliable predictors for evaluating therapeutic efficacy.

Radiomics is a burgeoning research field that focuses on the extraction of large-scale image data and their analysis via high-throughput mining of quantitative imaging features [3-6]. The field has gradually gained importance in oncology for its value in diagnostic, prognostic, and predictive accuracy [7]. Using medical imaging data for diagnosis and prognostication stems from the notion that tumors of a specific genotype display heterogeneous phenotypes and anatomic variations [8]. The application of radiomics involves the use of personalized image data that can be incorporated within the clinical decision-support system, enabling the construction of models that predict therapeutic outcomes [9]. As sophisticated tools for image analysis generate large amounts of data, machine learning (ML) has emerged as a powerful methodology for constructing precise predictive models, thus improving predictive performance [10].

In HCC, radiomics is particularly valuable because this cancer is most often diagnosed via dynamic threephase computed tomography (CT) scans, thus potentiating the use of image features to predict therapeutic responses. To the best of our knowledge, there have been no studies using CT-based 
radiomics to predict the effect of SBRT on HCC. Therefore, in addition to examining current evidence of SBRT in HCC, we aimed to propose a radiomics-based ML strategy for the prediction of local response (LR) after SBRT.

\section{Methods}

\section{Literature search}

A systematic online search using electronic databases was conducted by two independent medical doctors (Y.H.D. and P.C.S.). If there was an inconsistent selection and lack of agreement, another medical doctor (C.H.L.) made the final judgment and decision. The three electronic databases MEDLINE (PubMed), EMBASE, and LILACS were searched for studies dating from January 1993 to December 2018. The Medical Subject Headings terms of stereotactic body radiotherapy, SBRT, hepatocellular carcinoma, and HCC were used. The search was limited to humans, the English language, and patient numbers > 100.

\section{Patients for Clinical Evaluation and Radiomic Analysis}

Clinical data of patients with HCC who received SBRT at our institution between 2007 and 2016 were retrospectively reviewed. For radiomic analysis, patients who underwent locoregional ablative treatments in the past were excluded. Enrolled patients had undergone dynamic CT before radiotherapy and followup CT to evaluate responses. Follow-up time was obtained from the date of treatment to the last outpatient visit, with a median follow-up time of 26.6 months. Because of the retrospective nature of this study, we obtained approval from our Institutional Review Board for a waiver of informed consent (IRB number: 1-107-05-016, analysis of treatment outcome of patients with hepatic tumors).

\section{SBRT and clinical endpoints}

All patients were treated with SBRT using the Cyberknife image-guided radiosurgery system (Accuray, Sunnyvale, CA) as described previously [11-13]. The modified Response Evaluation Criteria in Solid Tumors (mRECIST) system was used to assess therapeutic responses [14]. Tumors achieving complete and partial responses, as per mRECIST criteria, were considered as having local control (LC). The LC that occurred during the first year after treatment was considered as a 1-year LC or within 1-year response (W1R) in our study. End response was defined as a complete/partial response achieved by the end of the follow-up period (EndR). Tumors within the radiotherapy field that did not show increase in size at the end of the follow-up period were considered to have attained in-field failure-free (IFFF). EndR and IFFF were used to evaluate two different aspects of clinical response, with the former aiming to identify the final responders and the latter to address progressive events. 


\section{Extraction of radiomic features}

Complete dynamic, multiphasic CT scans with contrast were retrieved individually in the Digital Imaging and Communications in Medicine (DICOM) format. All scans were performed with a tube voltage of 120 $k V p$ and a pitch of 0.984 . The images were then imported to the Computational Environment for Radiotherapy Research (CERR) platform based on MATLAB (MathWorks, Naticks, MA, 2017b) for radiation planning analysis. For each contrast-phase CT scan (arterial (A), portovenous (E), and delayed (D)), tumor regions were contoured by two experienced radiation oncologists (W.Y.H. and C.H.L.) and modified by another experienced radiologist (W.C.C.) to minimize delineation uncertainty. Each slice was resized to $128 \times 128$ voxels using bicubic interpolation $(0.8301 \times 0.8301 \times 3 \mathrm{~mm} 3$ for each voxel). The intensity distribution was standardized by histogram equalization. To reduce image noise and increase the sensitivity of radiomic analyses, a bin width of 25 Hounsfield units was used for discretization prior to global texture analysis [15]. Radiomic features were subsequently extracted and divided into 4 categories: first-order statistics, shape, global, and texture. For matrix-based texture features, different combinations of quantization (equal probability (Ep) and uniform quantization $(U)$ ) of gray levels $(8,16$, 32 , and 64) were performed [16]. Incorporating CT scans from each phase, the training datasets combined features from different CT phases, quantization methods, and gray levels, and were termed $\operatorname{AEp}(8,16,32$, and 64), $\operatorname{AU}(8,16,32$, and 64), $\operatorname{EEp}(8,16,32$, and 64), $\operatorname{EU}(8,16,32$, and 64), DEp $(8,16,32$, and 64$)$, and $\mathrm{DU}(8,16,32$, and 64$)$. Altogether, there were 41 tumors identified and 46 radiomic features prior further processing.

\section{Machine Learning Characterization}

\section{Data augmentation and adjustment of imbalanced data}

Oversampling with bootstrapping was used to expand our data population to 5000 samples. Synthetic Minority Oversampling Technique (SMOTE) was then used to adjust the imbalanced data. For the minority class, SMOTE is advantageous for making the decision region more general and improving the classifier performance [17].

All 41 identified tumors were used for radiomic analyses. 78\% (32/41) of the initial samples were extracted randomly and oversampled for training. The remaining $22 \%(9 / 41)$ was oversampled in a similar way for subsequent testing. The final cohort comprised 5201 training and 1301 testing samples, both of which were then balanced by SMOTE for positive and negative LRs.

\section{Feature selection}


The radiomic features extracted from the contoured region of each tumor were averaged and subsequently normalized across the cohort. To select the radiomic features, a regression method used to improve prediction accuracy by incorporating penalized estimation functions known as Least Absolute Shrinkage and Selection Operator (LASSO) was used [18]. LASSO started feature selection by tuning a parameter $(\lambda)$. During this process, most covariate coefficients were shrunk to zero, and the remaining features with non-zero coefficients were selected. For each training set, which included the training features with the corresponding training responses (i.e. W1R, ENdR, and IFFF), the features selected by LASSO were used to build the classifiers.

\section{Support Vector Machine (SVM) and Logistic Regression (LRG) classifiers}

With high prediction accuracy in various clinical settings [19, 20], SVM and LRG were adopted in our study to construct classifiers for LR. The SVM classifier deals with non-linear interaction and was used to discriminate whether a LR was achieved or not [21]. The penalty parameter $C$ was set to 1 to determine the tradeoff between fitting error and model complexity. Radial basis function was used as the kernel function in our SVM classifier. The LRG was used to predict the likelihood of positive LR, and a probability equal to 0.5 was set as the minimum threshold to determine the predicted class.

\section{Evaluation of model performance}

The model performance of SVM and LRG classifiers were evaluated for accuracy, sensitivity, and specificity after 10 -fold cross-validation in the training cohorts. F1 score was used to evaluate the model robustness in the testing cohorts. The classifier with the highest mean F1 scores for the three LRs was chosen as the candidate model. Receiver operating characteristic (ROC) curves were then used to assess the output quality with area under curve (AUC) and 5-fold cross-validation. All the functions used in our analyses were based on Python.

\section{Statistical analysis}

For the evaluation of radiomic data processing methods, the 50th percentile of accuracy was used as the threshold. The numbers of processing methods with an accuracy above the 50th percentile were identified and used for comparison of parameter robustness. Kaplan-Meier (KM) analysis with log-rank test was used to evaluate the effect of therapeutic response on survival. P value $<0.05$ was considered significant. All statistical analyses and survival calculation were performed in R.

\section{Results}




\section{Reported Clinical evidence for SBRT in HCC}

A total of 5 retrospective studies with $>100$ patients were selected (Table 1). To summarize, SBRT led to encouragingly high LC (1-year LC: $87 \%$ to $99 \%$ ) in HCC [1, 22-25]. Despite a reported lower overall survival (OS) from Bujuold et al., other studies showed high 1-year OS, ranging from $94 \%$ to $99 \%$. Four of the five studies used SBRT in small- to medium-sized HCC, and all studies included patients with Child-Pugh (CP) class A liver function as the majority. Only Nabavizadeh et al. investigated SBRT in CP class $\mathrm{C}$ patients (13\%) [25]. The dose fractionation scheme was variable, ranging from 24 Gray (Gy) in 6 fractions to 60 Gy in 3 fractions, resulting in a biological effective dose ranging from $33.6 \mathrm{~Gy}$ to $78 \mathrm{~Gy}$.

\section{In-Patient Demographics}

Overall, 172 patients receiving SBRT for HCC were retrospectively reviewed in our institution. Patient information and therapeutic outcomes are summarized in Table 2. The median tumor size was $5.4 \mathrm{~cm}$, ranging from $0.8 \mathrm{~cm}$ to as large as $20.1 \mathrm{~cm}$. There were $76.7 \% \mathrm{CP}$ class A patients and $23.3 \% \mathrm{CP}$ class $\mathrm{B}$ patients in the overall cohort. In this cohort, $36.6 \%$ patients presented with portal vein thrombosis (PVT). Patients in the radiomic cohort showed similar characteristics with those in the overall cohort. The median tumor size was $5 \mathrm{~cm}$, with the largest treated tumor being $13 \mathrm{~cm}$. There were $84.8 \% \mathrm{CP}$ class A patients and $15.2 \%$ CP class B patients. Out of the 33 patients, $21.2 \%$ showed PVT. The radiation dose ranges were also similar between the two cohorts (25-65 Gy in 4-6 fractions in the overall cohort; 32-60 Gy in 4-6 fractions in the radiomic cohort).

\section{Construction and Evaluation of the Predictive Models}

Accounting for the three types of LR, a total of 72 datasets were analyzed. Using SVM, classifiers with the highest accuracy (98.7\%) were built from features processed in A phase with Ep quantization and 32 gray levels (AEp32) for W1R (Figure 1A). For EndR, features processed in E phase with Ep quantization and 8 gray levels (EEp8) had the highest accuracy (99.3\%, 95\% confidence interval [Cl] = 91.2-99.5\%); and for IFFFR, features processed in A phase with Ep quantization and 16 gray levels (AEp16) corresponded to the highest accuracy $(99.7 \%, 95 \% \mathrm{Cl}=99.5-99.8 \%)$. When we looked at sensitivity and specificity, CT scans in the $A$ and $D$ phases generally presented with higher sensitivity and specificity than in the $E$ phase (Supplementary Figure 1A). Interestingly, AEp8 exhibited the highest sensitivity $(98.2 \%, 95 \% \mathrm{Cl}=$ 97.9-99.0 \%) for W1R and the highest specificity $(76.5 \%, 95 \% \mathrm{Cl}=72.1-77.3 \%)$ for EndR. However, the specificity was generally low for IFFF in all datasets.

When examining the processing sources and methods, we identified roughly similar distribution of three phases for the three LRs in the 50th percentile of accuracy (Figure 1B). Moreover, classifiers with higher accuracy were mostly constructed by features with 8,16 , or 32 gray levels, not 64 gray levels. For the quantization methods, features processed with Ep quantization corresponded to higher accuracy for W1R 
$(n=9)$, compared with those processed with $U$ quantization $(n=3)$. In the testing cohort, dataset processed in the A phase with Ep quantization and 8 gray levels (AEp8) exhibited the highest mean F1 scores for the three LRs (0.7995) (Figure 1C). In separate conditions, Ep quantization and 8 gray levels in the A phase had the highest cumulative F1 scores compared to other parameters (Figure 1D). Based on the above findings, AEp8 was chosen as the candidate model. ROC curve was plotted, and we observed the AUC for IFFF was the highest (AUC $=99.2 \%, 95 \% \mathrm{Cl}=99.0-93.2 \%$ ), followed by EndR (AUC $=96.3 \%$, $95 \% \mathrm{Cl}=96.0-96.9 \%$ ) and $\mathrm{W} 1 \mathrm{R}$ in AEp8 (AUC $=92.1 \%, 95 \% \mathrm{Cl}=91.8-93.4 \%$ ) (Figure 1E). For AEp8, the optimal $\lambda$ for LASSO was 0.00045 and the most potential feature for W1R was Long Run High Gray-Level Emphasis (LRHGE, coefficient $=-5.135$ ). For EndR and IFFF, Long Zone High Gray-Level Emphasis (LZLGE, $\lambda=0.00039$, coefficient $=7.207$ ) and Long Run Low Gray-Level Emphasis (LRLGE, $\lambda=0.00035$, coefficient $=-3.587$ ) were the most predictive features, respectively.

For LRG, the classifiers with the highest accuracy for W1R, EndR, and IFFF were built from DU32 (98.2\%, 95\% Cl = 97.6-98.5\%), DEp64 (98.1\%, 95\% Cl = 97.6-99.0\%) and EU64 (99.5\%, 95\% Cl = 99.1-99.7\%)

(Figure 2A). We found no datasets presented with both high sensitivity and specificity for the three LRs (Supplementary Figure 1B). Like SVM, more E phases were noted in the 50th percentile of accuracy for EndR $(n=7)$; and the distribution of accuracy for Ep and $U$ quantization methods demonstrated greater discrepancy, as compared to SVM (Figure 2B). In the testing cohort, AEp64 was found to outperform other datasets in terms of LR prediction (mean F1 score for the three LRs $=0.7783$ ), even though the Ep quantization or 8 gray levels had higher cumulative F1 scores (Figure 2C and 2D). The ROC curve for AEp64 revealed that IFFF had the highest AUC $(99.2 \%, 95 \% \mathrm{Cl}=98.7-99.6 \%)$, whereas EndR exhibited the lowest AUC (86.1\%, 95\% Cl = 85.4-88.2\%) (Figure 2E). In AEp64, the most predictive feature were GrayLevel Non-Uniformity (GLN, $\lambda=0.00046$, coefficient $=12.566$ ), Long Gray-Level Run Emphasis (LGRE, $\lambda=$ 0.000395 , coefficient $=7.019)$, and LRHGE $(\lambda=0.00036$, coefficient $=-7.294)$ for W1R, EndR, and IFFF, respectively.

\section{Association of W1R with 1-year OS}

Patients with W1R had significantly higher survival $(P<0.001)$ (Figure 3$)$. The patients with W1R had a median survival of 27.4 months, compared to 8 months in patients without W1R. This result suggested that achieving W1R was associated with better survival probability.

\section{Discussion}

Despite great therapeutic outcomes and advances in radiation techniques, the treatment of HCC using SBRT remains challenging. The benefit of SBRT is stronger for CP class A patients, who can receive higher radiation doses and more aggressive fractionation schedules with lower toxicity [1, 22, 26, 23-25], than for CP class B patients $[27,26]$. Moreover, several studies also reported a clear survival benefit in CP class A compared to CP class $B[27,26,28,24]$. Therefore, the balance of benefits and risks should be carefully evaluated. In our study, patients undergoing SBRT were mostly in the CP class A $(76.7 \%$, 
Table 1). However, there were still 23.3\% CP class B patients. The latter had poorer liver function and thus the prescription of a higher dose was limited. Additionally, as high as $40.7 \%$ patients with W1R had PVT, which further reduced the chance of survival benefit $[29,30]$. Nonetheless, SBRT is still considered to be of therapeutic benefit for patients with PVT [31]. In the evaluable 53 cases of PVT, EndR was achieved in 42 cases $(79.2 \%)$, which represented a higher percentage than in patients who received Sorafenib alone (7\% based on $\mathrm{mRECIST}$ ) in a comparable study [32]. After a median follow-up of 32.8 months, the 1-year LC in the overall cohort was $85.4 \%$. This relatively lower LC could be explained by various definitions of LC across studies [1, 22, 26, 23-25]. In our analysis, only those having at least a partial response were considered as achieving LC. In a similar cohort in Taiwan, the reported 1-year in-field control was 85.3\% [33]. Even though this finding was quite similar to our result, their LC was defined as the absence of new lesion or increase in tumor size, which was less strict compared to our definition. Therefore, our result might be better and still comparable to the literature.

Scorsetti et al. used a similar definition of LC to that in our study and found that higher LC contributed to higher OS in HCC patients after SBRT [28]. In support of this, patients with W1R were shown in our study to have significantly longer survival than those without W1R (Fig. 3). Therefore, achieving LC seems to be of great clinical significance. In several previous reports, LC appears to be determined by tumor size [34, 28]. However, as aggressive tumors tend to exhibit increased intratumoral heterogeneity, the sole use of tumor size in predicting LR appears insufficient and inappropriate [35-37]. In addition to histologic and genomic study, radiomics is another non-invasive approach that enables a spatiotemporal and quantitative measurement of tumoral heterogeneity [38]. In the current study, 26 out of 46 features were based on the matrix manipulation, which were related to the quantitative description of size and intensity variations of the connected sub-regions. The most predictive features selected by LASSO for the three LRs were LRHGE, LZLGE, and LRLGE, respectively. These metrics quantify the heterogeneity in size and intensity within tumor volumes in CT images, which was in agreement with a previous study describing the significance of Gray-Level Uniformity for prognosis in HCC patients receiving radiotherapy [39]. These features are related to the spatial correlation with emphasis on gray levels, possibly capturing the intratumoral heterogeneity. However, the value of these features is attributed to the type of CT scans and the preprocessing methods. In our study, the features extracted from the A phase had higher mean F1 scores in either SVC or LRG classifiers. This finding is in line with the fact that the development of HCC tumor frequently involves neovascularization of unpaired arteries without associated portal tracts $[40,41]$ and consequently that contrast enhancement in the A phase with early wash-out in the $E$ phase is a widely accepted diagnostic criteria. Therefore, features in the A phase are believed to be much more informative, potentiating the prediction for LR. Unlike in other studies [42, 43], we used two quantization methods and identified that Ep quantization with 8 and 64 gray levels performed better on the A phase CT scans. Ep quantization method attempts to define a decision threshold in the tumor volume while maintaining the same number of voxels after quantization [16]. Ep quantization and 8 gray levels showed higher cumulative F1 scores individually in the A phase in the SVM and LRG classifiers (Fig. 1D). The SVM classifier based on AEp8 also exhibited the highest mean F1 score for the three LRs, suggesting the feasibility of these parameters for LR prediction. 
Even though we hereby proposed a radiomics-based ML strategy for SBRT in HCC, some limitations of current predictive models still need to be addressed prior to clinical application. First, the sample size was small in the initial dataset. Although we used an oversampling technique, the augmented data retained the intrinsic characteristics of the small number of original tumors, restricting its general utility. An alternative is to use image augmentation, generating large number of tumor images for training. However, this strategy also suffers from the similar limitation. Second, the tumors were not segmented automatically, thus the uncertainty of the peripheral regions might be increased. Future development of tumor segmentation out of normal liver could help refine this procedure. Furthermore, we did not adopt image filtering such as Laplacian or Gaussian filters, which could have enhanced the reproducibility of feature extraction. Finally, since low specificity was consistently observed for SVM and LRG, IFFF seemed poorly defined and required further elucidation. Once more patients are included with clearly defined target response, we believe that our model will exhibit some improved performance in the future.

\section{Conclusions}

In conclusion, this is the first study to propose a radiomics-based predictive model for SBRT efficacy in patients with HCC. The findings warrant further studies in larger populations to confirm the feasibility of using our radiomics-based model in the clinic.

\section{Abbreviations}

SBRT: Stereotactic body radiotherapy; HCC: hepatocellular carcinoma; ML: machine learning; LC: local response; SVM: Support Vector Machine; CT: computed tomography; Ep: equal probability ; W1R : local response within 1 year; EndR: local response at the end of follow-up; IFFF: in-field failure-free; AUC: are under the curve; mRECIST: modified Response Evaluation Criteria in Solid Tumors; DICOM: Digital Imaging and Communications in Medicine; CERR: Computational Environment for Radiotherapy Research; SMOTE: Synthetic Minority Oversampling Technique; LASSO: Least Absolute Shrinkage and Selection Operator; LRG: logistic regression; ROC: Receiver operating characteristic; KM: Kaplan-Meier; OS: overall survival; CP: Child-Pugh; PVT: portal vein thrombosis; LRHGE: Long Run High Gray-Level Emphasis; GLN: Gray-Level Non-Uniformity; LGRE: Long Gray-Level Run Emphasis

\section{Declarations}

\section{Ethics approval and consent to participate}

This study was approved by the Research Ethics Committee of Tri-Service General Hospital (IRB number: 1-107-05-016) with a waiver of informed consent.

\section{Consent for publication}




\section{Availability of data and materials}

The datasets generated and analyzed during the current study are available from the corresponding author on reasonable request.

\section{Competing interests}

The authors declare no conflicts of interest

\section{Funding}

This study was supported in part by the study projects of National Defense Medical Center and Kaohsiung Armed Forces General Hospital (KAFGH 108-41 \& KAFGH-E-109048)

\section{Authors' contributions}

Conceptualization, Y.H.D. and P.C.S.; methodology, Y.H.D.; software, Y.H.D. and J.F.Y.; validation, W.Y.H., C.H.L. and S.J.T.; formal analysis, Y.H.D.; investigation, P.C.S.; resources, Y.H.D. and P.C.S.; data curation, W.C.C. and H.L.C.; writing-original draft preparation, Y.H.D.; writing-review and editing, W.Y.H., C.H.L., C.S.L and S.J.T.; visualization, Y.H.D.; supervision, W.Y.H. and S.J.T.; project administration, W.Y.H.

\section{Acknowledgements}

The authors thank the Cancer Registry Group of Tri-Service General Hospital for the clinical data support.

\section{References}

1. Bujold A, Massey CA, Kim JJ, Brierley J, Cho C, Wong RK, et al. Sequential phase I and II trials of stereotactic body radiotherapy for locally advanced hepatocellular carcinoma. Journal of clinical oncology : official journal of the American Society of Clinical Oncology. 2013 May 1;31(13):1631-9.

2. Wahl DR, Stenmark MH, Tao Y, Pollom EL, Caoili EM, Lawrence TS, et al. Outcomes After Stereotactic Body Radiotherapy or Radiofrequency Ablation for Hepatocellular Carcinoma. J Clin Oncol. 2016 Feb 10;34(5):452-9. 
3. El Naqa I, Grigsby P, Apte A, Kidd E, Donnelly E, Khullar D, et al. Exploring feature-based approaches in PET images for predicting cancer treatment outcomes. Pattern Recognit. 2009 Jun 1;42(6):1162-71.

4. Gillies RJ, Anderson AR, Gatenby RA, Morse DL. The biology underlying molecular imaging in oncology: from genome to anatome and back again. Clin Radiol. 2010 Jul;65(7):517-21.

5. Kumar V, Gu Y, Basu S, Berglund A, Eschrich SA, Schabath MB, et al. Radiomics: the process and the challenges. Magn Reson Imaging. 2012 Nov;30(9):1234-48.

6. Lambin P, Rios-Velazquez E, Leijenaar R, Carvalho S, van Stiphout RG, Granton P, et al. Radiomics: extracting more information from medical images using advanced feature analysis. Eur $\mathrm{J}$ Cancer. 2012 Mar;48(4):441-6.

7. Lambin P, Leijenaar RTH, Deist TM, Peerlings J, de Jong EEC, van Timmeren J, et al. Radiomics: the bridge between medical imaging and personalized medicine. Nat Rev Clin Oncol. 2017 Dec;14(12):749-62.

8. Aerts HJ, Velazquez ER, Leijenaar RT, Parmar C, Grossmann P, Carvalho S, et al. Decoding tumour phenotype by noninvasive imaging using a quantitative radiomics approach. Nat Commun. 2014 Jun 3;5:4006.

9. Lambin P, van Stiphout RG, Starmans MH, Rios-Velazquez E, Nalbantov G, Aerts HJ, et al. Predicting outcomes in radiation oncology--multifactorial decision support systems. Nat Rev Clin Oncol. 2013 Jan;10(1):27-40.

10. Parmar C, Grossmann P, Bussink J, Lambin P, Aerts H. Machine Learning methods for Quantitative Radiomic Biomarkers. Sci Rep. 2015 Aug 17;5:13087.

11. Huang WY, Jen YM, Lee MS, Chang LP, Chen CM, Ko KH, et al. Stereotactic body radiation therapy in recurrent hepatocellular carcinoma. Int J Radiat Oncol Biol Phys. 2012 Oct 1;84(2):355-61.

12. Lo CH, Liu MY, Lee MS, Yang JF, Jen YM, Lin CS, et al. Comparison Between Child-Turcotte-Pugh and Albumin-Bilirubin Scores in Assessing the Prognosis of Hepatocellular Carcinoma After Stereotactic Ablative Radiation Therapy. Int J Radiat Oncol Biol Phys. 2017 Sep 1;99(1):145-52.

13. Shen PC, Chang WC, Lo CH, Yang JF, Lee MS, Dai YH, et al. Comparison of Stereotactic Body Radiation Therapy and Transarterial Chemoembolization for Unresectable Medium-Sized Hepatocellular Carcinoma. Int J Radiat Oncol Biol Phys. 2019 Jun 5.

14. Llovet JM, Di Bisceglie AM, Bruix J, Kramer BS, Lencioni R, Zhu AX, et al. Design and endpoints of clinical trials in hepatocellular carcinoma. J Natl Cancer Inst. 2008 May 21;100(10):698-711.

15. Huynh E, Coroller TP, Narayan V, Agrawal V, Hou Y, Romano J, et al. CT-based radiomic analysis of stereotactic body radiation therapy patients with lung cancer. Radiother Oncol. 2016 Aug;120(2):25866.

16. Vallieres M, Freeman CR, Skamene SR, El Naqa I. A radiomics model from joint FDG-PET and MRI texture features for the prediction of lung metastases in soft-tissue sarcomas of the extremities. Phys Med Biol. $2015 \mathrm{Jul}$ 21;60(14):5471-96.

17. Nitesh V. C. KWB, Lawrence O. H. et al. SMOTE: Synthetic Minority Over-sampling Technique. J Artif Intell Res. 2002;16:321-57. 
18. Tibshirani R. Regression shrinkage and selection via the lasso: a retrospective. J R Statist Soc B. 2011;73:273-82.

19. Yahya N, Ebert MA, Bulsara M, House MJ, Kennedy A, Joseph DJ, et al. Statistical-learning strategies generate only modestly performing predictive models for urinary symptoms following external beam radiotherapy of the prostate: A comparison of conventional and machine-learning methods. Med Phys. 2016 May;43(5):2040.

20. Lee HC, Yoon HK, Nam K, Cho YJ, Kim TK, Kim WH, et al. Derivation and Validation of Machine Learning Approaches to Predict Acute Kidney Injury after Cardiac Surgery. J Clin Med. 2018 Oct $3 ; 7(10)$.

21. Gultepe E, Green JP, Nguyen H, Adams J, Albertson T, Tagkopoulos I. From vital signs to clinical outcomes for patients with sepsis: a machine learning basis for a clinical decision support system. J Am Med Inform Assoc. 2014 Mar-Apr;21(2):315-25.

22. Sanuki N, Takeda A, Oku Y, Mizuno T, Aoki Y, Eriguchi T, et al. Stereotactic body radiotherapy for small hepatocellular carcinoma: a retrospective outcome analysis in 185 patients. Acta Oncol. 2014 Mar;53(3):399-404.

23. Su TS, Liang P, Lu HZ, Liang J, Gao YC, Zhou Y, et al. Stereotactic body radiation therapy for small primary or recurrent hepatocellular carcinoma in 132 Chinese patients. J Surg Oncol. 2016 Feb;113(2):181-7.

24. Jeong Y, Jung J, Cho B, Kwak J, Jeong C, Kim JH, et al. Stereotactic body radiation therapy using a respiratory-gated volumetric-modulated arc therapy technique for small hepatocellular carcinoma. BMC Cancer. 2018 Apr 13;18(1):416.

25. Nabavizadeh N, Waller JG, Fain R, 3rd, Chen Y, Degnin CR, Elliott DA, et al. Safety and Efficacy of Accelerated Hypofractionation and Stereotactic Body Radiation Therapy for Hepatocellular Carcinoma Patients With Varying Degrees of Hepatic Impairment. Int J Radiat Oncol Biol Phys. 2018 Mar 1;100(3):577-85.

26. Lasley FD, Mannina EM, Johnson CS, Perkins SM, Althouse S, Maluccio M, et al. Treatment variables related to liver toxicity in patients with hepatocellular carcinoma, Child-Pugh class $A$ and $B$ enrolled in a phase 1-2 trial of stereotactic body radiation therapy. Pract Radiat Oncol. 2015 Sep-Oct;5(5):e443e49.

27. Cardenes HR, Price TR, Perkins SM, Maluccio M, Kwo P, Breen TE, et al. Phase I feasibility trial of stereotactic body radiation therapy for primary hepatocellular carcinoma. Clinical \& translational oncology : official publication of the Federation of Spanish Oncology Societies and of the National Cancer Institute of Mexico. 2010 Mar;12(3):218-25.

28. Scorsetti M, Comito T, Cozzi L, Clerici E, Tozzi A, Franzese C, et al. The challenge of inoperable hepatocellular carcinoma (HCC): results of a single-institutional experience on stereotactic body radiation therapy (SBRT). J Cancer Res Clin Oncol. 2015 Jul;141(7):1301-9.

29. Schoniger-Hekele M, Muller C, Kutilek M, Oesterreicher C, Ferenci P, Gangl A. Hepatocellular carcinoma in Central Europe: prognostic features and survival. Gut. 2001 Jan;48(1):103-9. 
30. Yeh SA, Chen YS, Perng DS. The role of radiotherapy in the treatment of hepatocellular carcinoma with portal vein tumor thrombus. J Radiat Res. 2015 Mar;56(2):325-31.

31. Shui $Y, Y u$ W, Ren $X$, Guo Y, Xu J, Ma T, et al. Stereotactic body radiotherapy based treatment for hepatocellular carcinoma with extensive portal vein tumor thrombosis. Radiat Oncol. 2018 Sep 25;13(1):188.

32. Gavanier M, Ayav A, Sellal C, Orry X, Claudon M, Bronowicki JP, et al. CT imaging findings in patients with advanced hepatocellular carcinoma treated with sorafenib: Alternative response criteria (Choi, European Association for the Study of the Liver, and modified Response Evaluation Criteria in Solid Tumor (mRECIST)) versus RECIST 1.1. Eur J Radiol. 2016 Jan;85(1):103-12.

33. Que J, Kuo HT, Lin LC, Lin KL, Lin CH, Lin YW, et al. Clinical outcomes and prognostic factors of cyberknife stereotactic body radiation therapy for unresectable hepatocellular carcinoma. BMC Cancer. 2016 Jul 12;16:451.

34. Kang JK, Kim MS, Cho CK, Yang KM, Yoo HJ, Kim JH, et al. Stereotactic body radiation therapy for inoperable hepatocellular carcinoma as a local salvage treatment after incomplete transarterial chemoembolization. Cancer. 2012 Nov 1;118(21):5424-31.

35. Fidler IJ. Critical factors in the biology of human cancer metastasis: twenty-eighth G.H.A. Clowes memorial award lecture. Cancer Res. 1990 Oct 1;50(19):6130-8.

36. Yokota J. Tumor progression and metastasis. Carcinogenesis. 2000 Mar;21(3):497-503.

37. Campbell PJ, Yachida S, Mudie LJ, Stephens PJ, Pleasance ED, Stebbings LA, et al. The patterns and dynamics of genomic instability in metastatic pancreatic cancer. Nature. 2010 Oct 28;467(7319):1109-13.

38. Sanduleanu S, Woodruff HC, de Jong EEC, van Timmeren JE, Jochems A, Dubois L, et al. Tracking tumor biology with radiomics: A systematic review utilizing a radiomics quality score. Radiother Oncol. 2018 Jun;127(3):349-60.

39. Cozzi L, Dinapoli N, Fogliata A, Hsu WC, Reggiori G, Lobefalo F, et al. Radiomics based analysis to predict local control and survival in hepatocellular carcinoma patients treated with volumetric modulated arc therapy. BMC cancer. 2017 Dec 6;17(1):829.

40. Nakashima O, Sugihara S, Kage M, Kojiro M. Pathomorphologic characteristics of small hepatocellular carcinoma: a special reference to small hepatocellular carcinoma with indistinct margins. Hepatology. 1995 Jul;22(1):101-5.

41. International Consensus Group for Hepatocellular NeoplasiaThe International Consensus Group for Hepatocellular N. Pathologic diagnosis of early hepatocellular carcinoma: a report of the international consensus group for hepatocellular neoplasia. Hepatology. 2009 Feb;49(2):658-64.

42. Chen S, Zhu Y, Liu Z, Liang C. Texture analysis of baseline multiphasic hepatic computed tomography images for the prognosis of single hepatocellular carcinoma after hepatectomy: A retrospective pilot study. Eur J Radiol. 2017 May;90:198-204.

43. Zhou Y, He L, Huang Y, Chen S, Wu P, Ye W, et al. CT-based radiomics signature: a potential biomarker for preoperative prediction of early recurrence in hepatocellular carcinoma. Abdominal radiology. 
2017 Jun;42(6):1695-704.

\section{Tables}

able 1 Large series for stereotactic body radiotherapy in hepatocellular carcinoma

\begin{tabular}{|c|c|c|c|c|c|c|c|c|}
\hline uthor & Year & Patients & Tumor Size/Volume & $\mathrm{CP}$ class & Follow-up time (month) & Dose $^{a}$ & 1-year LC & 1-year OS \\
\hline ujuold et al ${ }^{1}$ & 2013 & 102 & $1-1913 \mathrm{~mL}$ & $\mathrm{~A}$ & 31 & $24-54 \mathrm{~Gy} / 6 \mathrm{fx}$ & $87 \%$ & $55 \%$ \\
\hline annuki et al ${ }^{22}$ & 2014 & 185 & $1.6-65 \mathrm{~mL}$ & $\begin{array}{l}\text { A }(85 \%), \\
\text { B (15\%) }\end{array}$ & 23 & $35-45$ Gy/ $5 \mathrm{fx}$ & $99 \%$ & $95 \%$ \\
\hline u et al 23 & 2016 & 132 & $1.1-5 \mathrm{~cm}$ & $\begin{array}{l}\text { A (86\%), } \\
\text { В (14\%) }\end{array}$ & 8 & $42-46$ Gy $/ 3 \mathrm{fx}$ & $91 \%$ & $94 \%$ \\
\hline $\begin{array}{l}\text { abavizadeh et al } \\
\text { : }\end{array}$ & 2018 & 146 & NR & $\begin{array}{l}\text { A }(46 \%), \\
\text { B }(41 \%), \\
\text { C }(13 \%)\end{array}$ & 23 & $50 \mathrm{~Gy} / 5 \mathrm{fx}$ & $97 \%$ & NR \\
\hline :ong et al & 2018 & 119 & $1.7 \mathrm{~cm}$ & $\begin{array}{l}\text { A }(91 \%) \\
\text { В }(9 \%)\end{array}$ & 26 & $30-60$ Gy/3 fx & $99 \%$ & $99 \%$ \\
\hline
\end{tabular}

radiation dose described in these studies; CP class: Child-Pugh class; Gy: Gray; fx: fractions; LC: local control; OS: overall survival; NR: not sported 
Table 2 Patient demographics for overall and radiomic cohorts

\begin{tabular}{|c|c|c|}
\hline Baseline features & Overall cohort & Radiomic cohort \\
\hline Patient number & 172 & 33 \\
\hline Tumor size (median) & $5.4 \mathrm{~cm}(0.8-20.1 \mathrm{~cm})$ & $5 \mathrm{~cm}(1.8-13 \mathrm{~cm})$ \\
\hline Age (S.D.) & $63 \pm 13$ & $68.5 \pm 12$ \\
\hline Sex & Male (76.7\%), Female (23.3\%) & Male $(63.6 \%)$, Female $(36.4 \%)$ \\
\hline CP class & A (76.7\%), B (23.3 \%) & $\mathrm{A}(84.8 \%), \mathrm{B}(15.2 \%)$ \\
\hline ECOG & 0 (47.7\%), 1 (38.4\%), 2 (13.9\%) & 0 (42.4\%), 1(39.3\%), 2 (18.3\%) \\
\hline PVT & $36.6 \%$ & $21.2 \%$ \\
\hline Dose & $25-65 \mathrm{~Gy} / 4-6 \mathrm{fx}$ & $32-60 \mathrm{~Gy} / 4-6 \mathrm{fx}$ \\
\hline Median F/u (month) & 32.8 & 26.6 \\
\hline \multicolumn{3}{|c|}{$\begin{array}{l}\text { S.D.: standard deviation; CP class: Child-Pugh class; ECOG: Eastern Cooperative Oncology Group; PVT: portal vein thrombosis; Gy: Gray; fx: } \\
\text { fractions; F/u: Follow-up }\end{array}$} \\
\hline
\end{tabular}

\section{Figures}


A

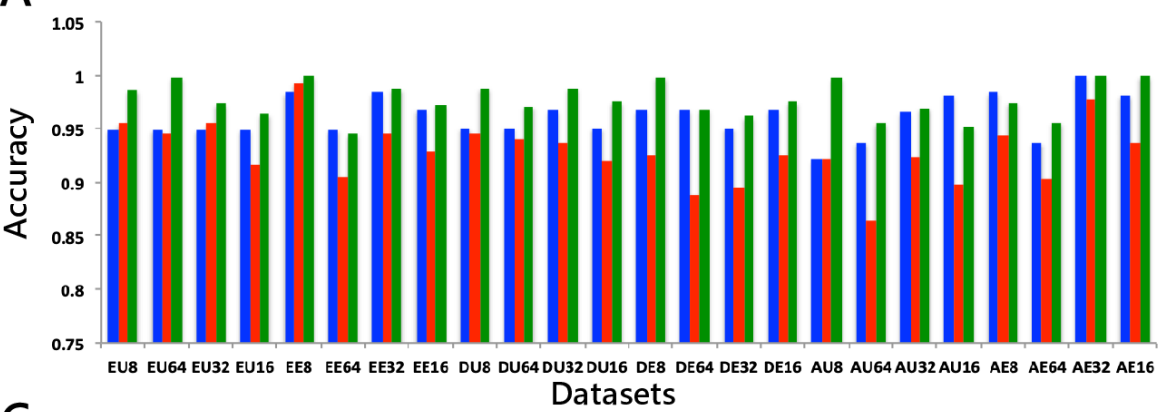

C

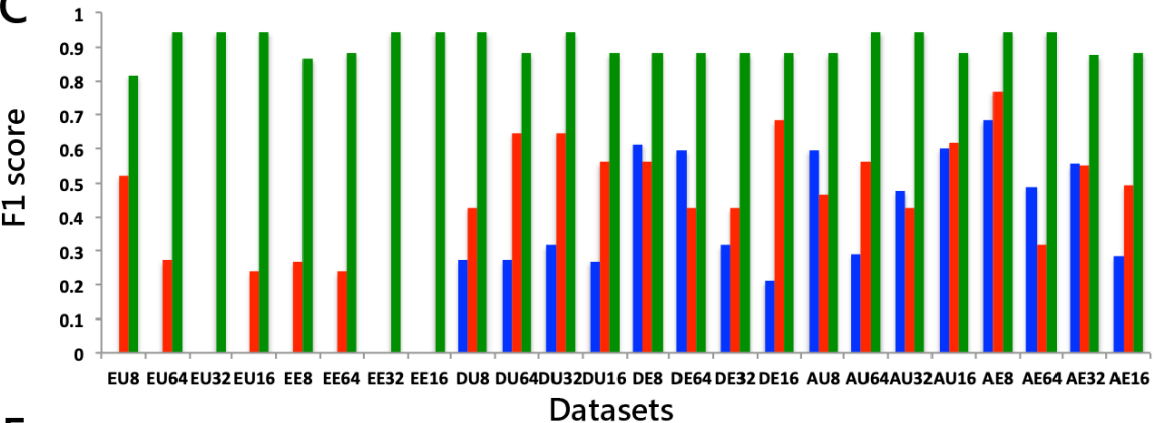

$E$

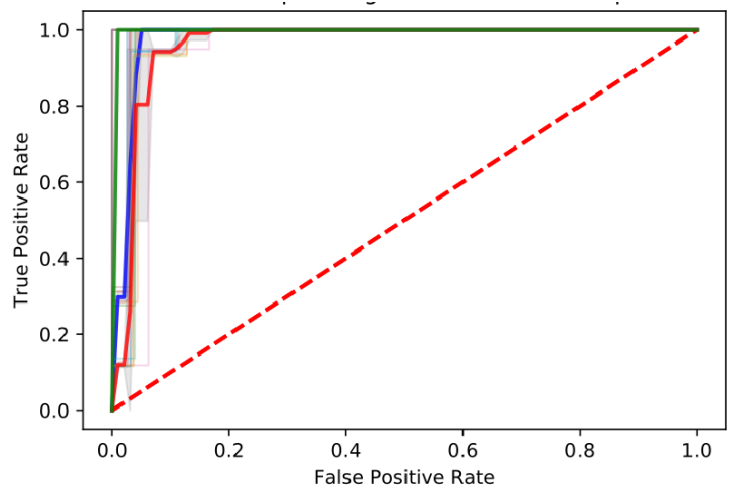

B
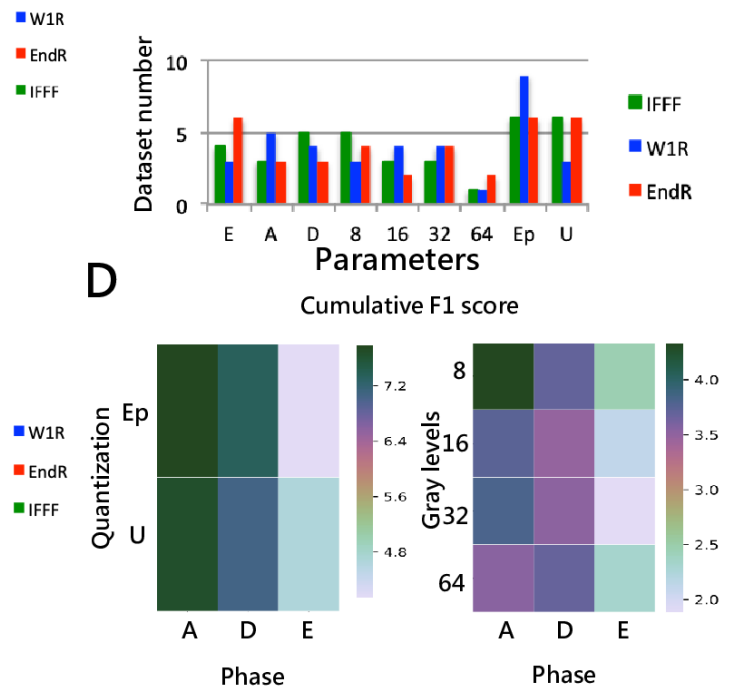

\section{Figure 1}

Model performance of Support Vector Machine (SVM) classifiers for different datasets. (A) Model accuracy in training cohort. (B) 50th percentile of accuracy for radiomic parameters. (C) F1 score in testing cohort. (D) Cumulative F1 score across 72 datasets for different phases, quantization methods and gray levels (E) Receiver operating characteristic (ROC) curve for AEp8. A: arterial phase; D: delayed phase; E: porto-venous phase; Ep: Equal probability quantization; U: Uniform quantization; W1R: within 1year response; EndR: End response; IFFF: in-field failure free. 
A

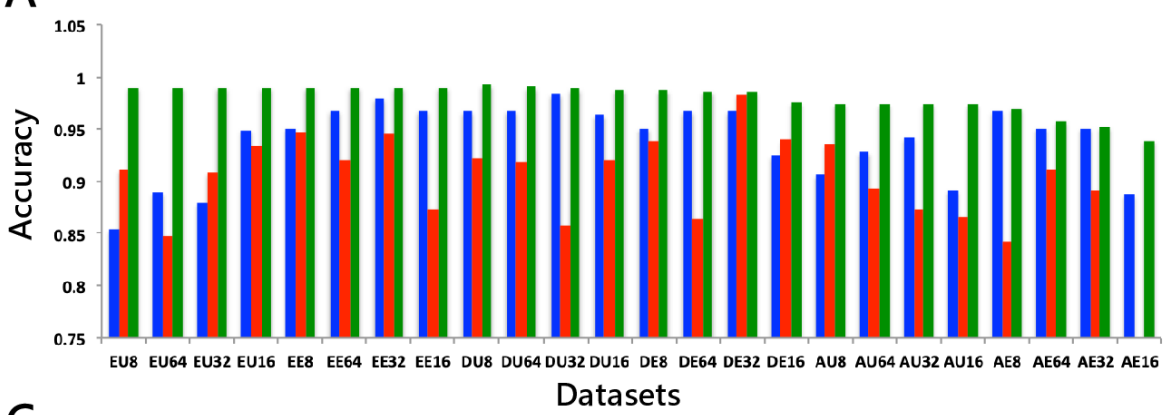

C

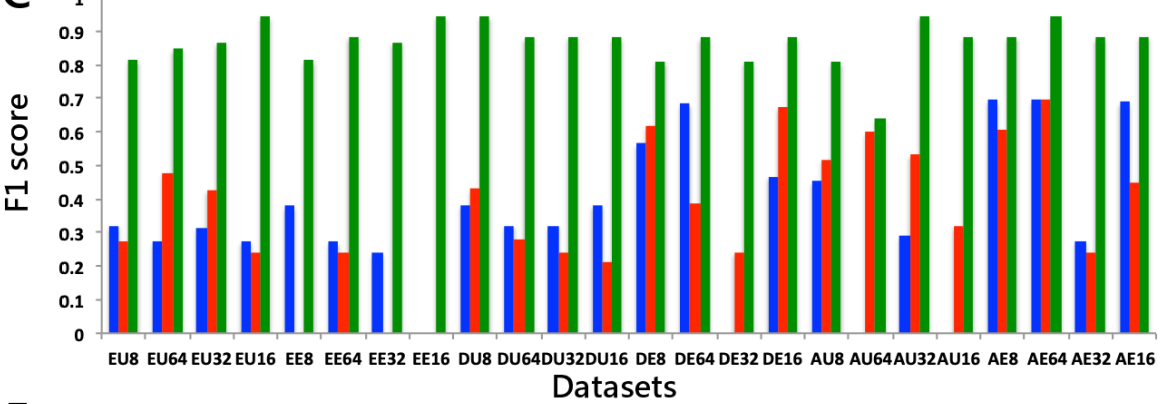

$E$

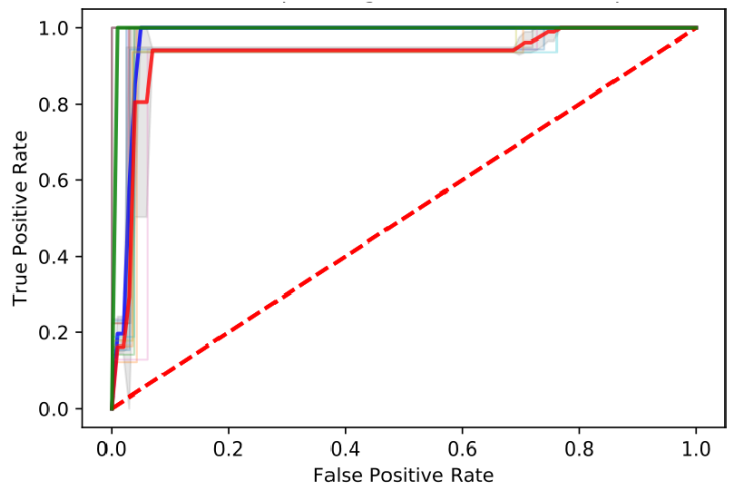

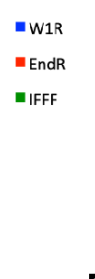

D
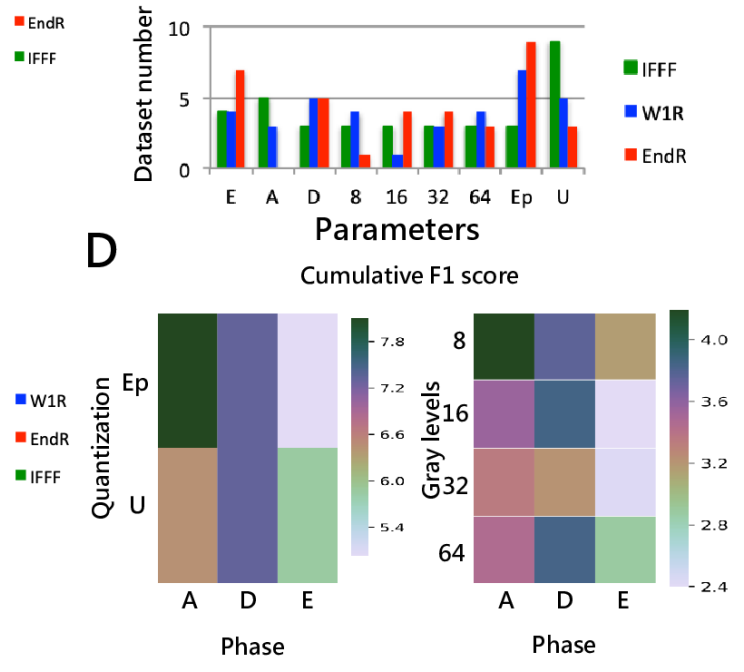

Parameters

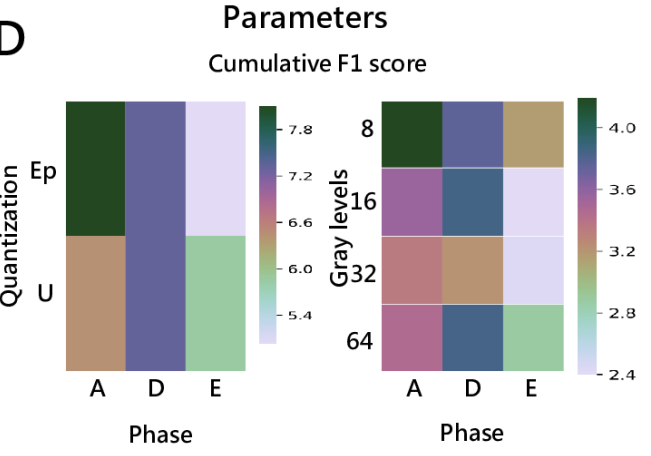

\section{Figure 2}

Model performance of Logistic Regression (LRG) classifiers for different datasets. (A) Model accuracy in training cohort. (B) 50th percentile of accuracy for radiomic parameters. (C) F1 score in testing cohort. (D) Cumulative F1 score across 72 datasets for different phases, quantization methods and gray levels $(E)$ Receiver operating characteristic (ROC) curve for AEp64. A: arterial phase; D: delayed phase; E: portovenous phase; Ep: Equal probability quantization; U: Uniform quantization; W1R: within 1-year response; EndR: End response; IFFF: in-field failure free. 

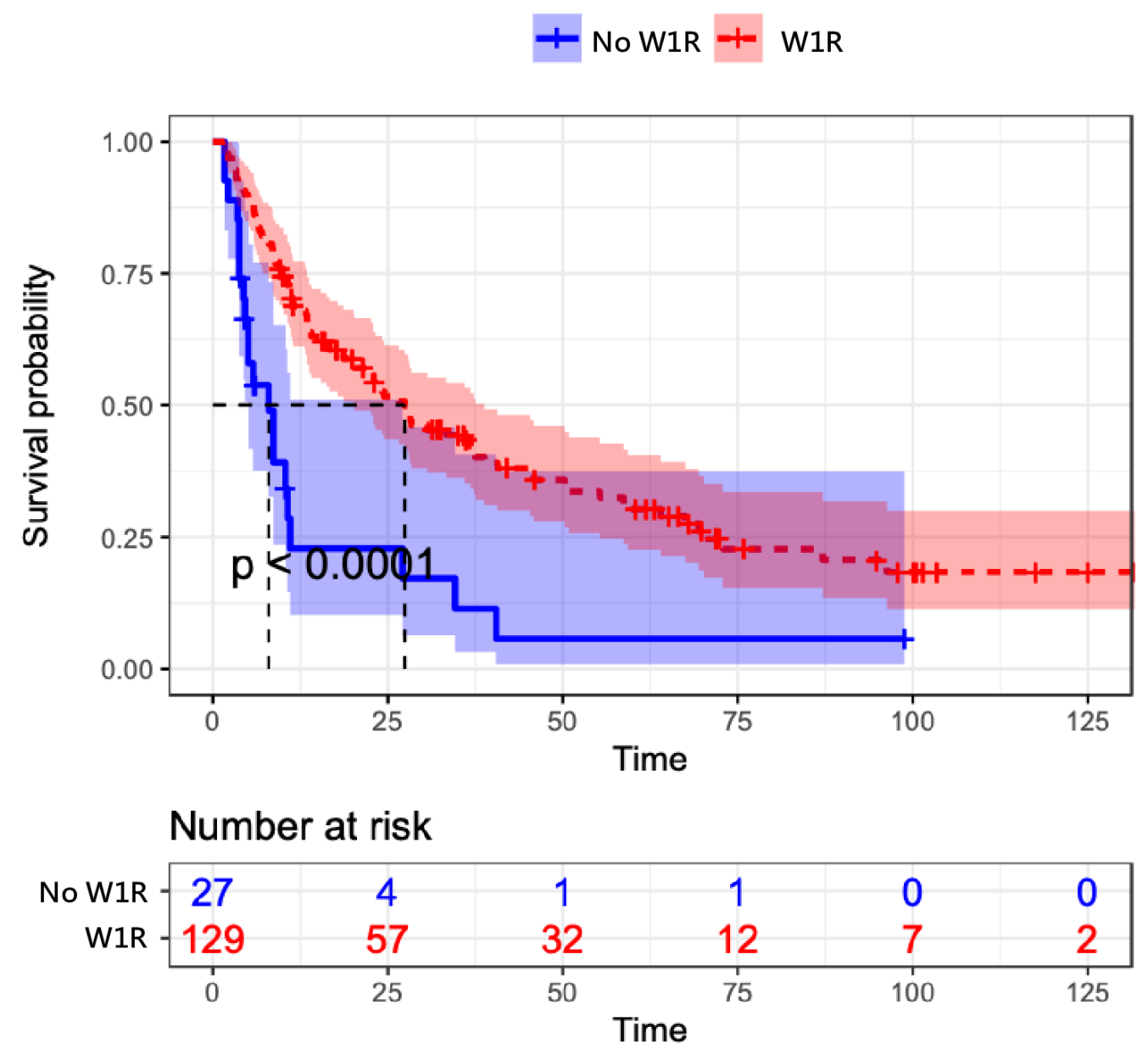

Figure 3

Kaplan-Meier plot for patients with or without 1-year response (W1R). 